\title{
CONVERGENCE OF CLOSED SUBSETS IN A TOPOLOGICAL SPACE
}

\author{
EDWARD G. EFFROS ${ }^{1}$
}

In some recent work, the author attempted to place a topology, or at least a Borel structure (see $[4, \S \$ 1-3]$ ) on the set of closed left ideals in a separable Banach algebra. Our approach was to introduce a notion of convergence for nets in the set $\mathrm{e}_{0}(X)$ of nonempty closed subsets of a topological space $X$ (see below, and $[3, \S 25]$ ). Unfortunately, as was pointed out to us by Calvin Moore (see Remark below), the convergence is generally not defined by a topology on $\mathfrak{C}_{0}(X)$. Nonetheless, there is a topology associated with the convergence, and the purpose of this note is to show that if $X$ is polonais (i.e., separable and metrizable by a complete metric), the generated Borel structure is standard. This will be applied to Banach algebras in a subsequent article.

If $F_{\alpha}$ is a net in $\mathrm{e}(X)=\mathfrak{C}_{0}(X) \cup\{\varnothing\}$, define lim inf $F_{\alpha}$ (lim sup $F_{\alpha}$ ) to be those $x$ in $X$ such that every neighborhood of $x$ is eventually (frequently) intersected by the $F_{\alpha}$ (we use the terminology of [2, p. 65]). These sets are closed, and if $F=\lim \inf F_{\alpha}=\lim \sup F_{\alpha}$, we say that $F_{\alpha}$ converges to $F, F$ is the limit of the $F_{\alpha}$, and we write $F_{\alpha} \rightarrow F$. If $\Sigma \subseteq \mathcal{C}(X)$, let $\bar{\Sigma}$ be the limits of all nets in $\Sigma$. Then $\{\varnothing\}^{-}$ $=\{\varnothing\}, \mathfrak{C}(X)^{-}=\mathfrak{e}(X),\left(\Sigma_{1} \cup \Sigma_{2}\right)^{-}=\bar{\Sigma}_{1} \cup \bar{\Sigma}_{2}$, but in general, $(\bar{\Sigma})^{-} \neq \bar{\Sigma}$. Defining a set $\Sigma$ to be convergence closed if $\bar{\Sigma}=\Sigma$, we obtain a topology on $\mathcal{e}(X)$. We call the relative topology and generated Borel structure on $\mathfrak{C}_{0}(X)$ the convergence topology and convergence Borel structure, respectively.

THEOREM. If $X$ is polonais, then the convergence Borel structure on $\mathfrak{C}_{0}(X)$ is standard.

Proof. As $X$ may be imbedded in a compact metric space (see [2, p. 125]), it is metrizable by a totally bounded metric $d$. Define a metric $\rho$ on $\mathfrak{e}_{0}(X)$ by

$$
\rho\left(F_{1}, F_{2}\right)=\max \left[\operatorname{lub}_{x_{1} \in F_{1}} d\left(x_{1}, F_{2}\right) \operatorname{lub}_{x_{2} \in F_{2}} d\left(x_{2}, F_{1}\right)\right],
$$

where in general, $d(x, F)=\operatorname{glb}_{y \in F} d(x, y)$, (see $[3, \S 15]$ for a discussion of this metric). Then we have:

Received by the editors June 8, 1964.

1 This work has been partially supported by the National Science Foundation under grant NSF GP1604. 
Lemma. $\left(\mathfrak{C}_{0}(X), \rho\right)$ is polonais.

Proof. Let $\bar{X}$ be the completion of $X$ with respect to $d$, and $\bar{\rho}$ the corresponding metric on $\mathfrak{C}_{0}(\bar{X})$. As $\bar{X}$ is compact, the same is true for $\left(\mathfrak{C}_{0}(\bar{X}), \bar{\rho}\right)($ see $[3, \S 15, \mathrm{IX}])$. Define $\iota: \mathfrak{C}_{0}(X) \rightarrow \mathfrak{C}_{0}(\bar{X})$ by $\iota(F)=\bar{F}$. It is readily verified that $\iota$ is an isometry, and that

$$
\iota\left(\mathfrak{e}_{0}(X)\right)=\left\{K \in \mathfrak{C}_{0}(\bar{X}): K \cap X \text { is dense in } K\right\} .
$$

It suffices to show that $\iota\left(\mathfrak{C}_{0}(X)\right)$ is a $G_{\delta}$ subset of $\mathfrak{e}_{0}(\bar{X})$ (see $[2$, p. 207]).

As $X$ is polonais, $X=\bigcap_{n=1}^{\infty} V_{n}$, where $V_{n}$ is open in $\bar{X}$. The Baire Category Theorem implies that if $K \in \mathrm{e}_{0}(\bar{X})$, then $K \cap X$ is dense in $K$ if and only if $K \cap V_{n}$ is dense in $K$ for all $n$, i.e.,

$$
\iota\left(\mathfrak{e}_{0}(X)\right)=\bigcap_{n=1}^{\infty}\left\{K \in \mathfrak{C}_{0}(\bar{X}): K \cap V_{n} \text { is dense in } K\right\} .
$$

If $K \cap V_{n}$ is not dense in $K$, there exists an $L \in \mathrm{e}_{0}(\bar{X})$ with $K \cap V_{n}$ $\subseteq L \subseteq K, L \neq K$. Letting $\Sigma_{n}$ be the complement of the quantity in curly brackets,

$$
\Sigma_{n}=\pi_{1}\left[\left\{(K, L) \in \mathfrak{C}_{0}(\bar{X}) \times \mathcal{C}_{0}(\bar{X}): K \cap V_{n} \subseteq L \subseteq K\right\}-\Delta\right],
$$

where $\pi_{1}: \mathfrak{C}_{0}(\bar{X}) \times \mathfrak{C}_{0}(\bar{X}) \rightarrow \mathfrak{C}_{0}(\bar{X})$ is the projection on the first coordinate, and $\Delta$ is the set of $(K, K)$ with $K \in \mathfrak{C}_{0}(\bar{X})$. Letting $S_{n}$ be the new quantity in curly brackets, $S_{n}$ is closed. For if $\left(K_{\alpha}, L_{\alpha}\right)$ is a net in $S_{n}$ converging to $(K, L) \in \mathfrak{e}_{0}(\bar{X}) \times \mathfrak{e}_{0}(\bar{X})$, say that $x \in K \cap V_{n}$. Then there is a net $x_{\alpha}$ with $x_{\alpha} \in K_{\alpha}$ and $d\left(x_{\alpha}, x\right) \rightarrow 0$. As $x \in V_{n}$, we eventually have $x_{\alpha} \in K_{\alpha} \cap V_{n} \subseteq L_{\alpha}$, hence $d\left(x, L_{\alpha}\right) \rightarrow 0$ and $x \in L$. A similar argument shows that $L \subseteq K$. Since the diagonal $\Delta$ is also closed, and $\mathfrak{e}_{0}(\bar{X}) \times \mathfrak{e}_{0}(\bar{X})$ is metrizable by a separable metric, $S_{n}-\Delta$ is $F_{\sigma}$, or as $\mathcal{C}_{0}(\bar{X})$ is compact, a countable union of compact sets. As $\pi_{1}$ is continuous, $\Sigma_{n}=\pi_{1}\left(S_{n}-\Delta\right)$ is $F_{\sigma}$, and

$$
\iota\left(\mathfrak{C}_{0}(X)\right)=\bigcap_{n=1}^{\infty}\left[\mathfrak{C}_{0}(\bar{X})-\Sigma_{n}\right]
$$

is $G_{\delta}$.

Proof of TheOREM (CONTINUEd). It follows that the metric Borel structure on $\mathfrak{C}_{0}(X)$ is standard. It is readily verified that if $F_{\alpha}$ is a net in $\mathfrak{e}_{0}(X)$ with $\rho\left(F_{\alpha}, F\right) \rightarrow 0$, then $F_{\alpha} \rightarrow F$. Thus the convergence topology is contained in the metric topology, and the convergence Borel structure is contained in the metric Borel structure. To show that the two structures coincide, it suffices to show that the convergence Borel structure is countably separated, i.e., there exists a countable family of convergence Borel sets such that no two points of 
$\mathfrak{C}_{0}(X)$ lie in the same members of the family (see [4, Theorem 3.2]). Let $x_{n}$ be dense in $X$, and define

$$
\Theta_{m n}=\left\{F \in \mathcal{C}_{0}(X): d\left(x_{n}, F\right) \geqq \frac{1}{m}\right\} .
$$

$\Theta_{m n}$ is convergence closed, as in general, if $F_{\alpha}$ is a net in $\mathfrak{C}_{0}(X)$ with $d\left(x, F_{\alpha}\right) \geqq \epsilon$, then $d\left(x, \lim \sup F_{\alpha}\right) \geqq \epsilon$. The sets $\Theta_{m n}$ separate points in $\mathfrak{C}_{0}(X)$, for suppose that $F_{1}, F_{2} \in \mathfrak{C}(X)$, and $F_{1}-F_{2} \neq \varnothing$. Select $y \in F_{1}-F_{2}$, and say that $d\left(y, F_{2}\right)=\epsilon$. Choose $m, n$ with $d\left(x_{n}, y\right)<1 / m$ $\leqq \epsilon / 2$. Then $d\left(x_{n}, F_{1}\right)<1 / m$ and $d\left(x_{n}, F_{2}\right) \geqq \epsilon-1 / m \geqq 1 / m$, i.e., $F_{1} \notin \Theta_{m n}$ and $F_{2} \in \Theta_{m n}$.

REMARK. Slightly extending some results of Calvin Moore (unpublished) we have

THEOREM. If $X$ Hausdorf, then convergence in $\mathrm{e}(X)$ is defined by a topology if and only if $X$ is locally compact. If these conditions hold, the topology is that defined by Fell in [1] (see below).

Sketching the proof, one first shows that for any $X, \mathrm{e}(X)$ is "compact" in the sense that any net in $\mathcal{C}(X)$ has a convergent subnet. In fact it is easily seen that any universal subnet (see [2, p. 81]) converges. Assume that $X$ is Hausdorff and convergence in $\mathfrak{e}(X)$ is defined by a topology. As a net can converge to only one point, the latter must be Hausdorff, and $\mathfrak{e}_{0}(X)$ is locally compact in the relative topology. As $X$ is Hausdorff, it readily follows that $I: X \rightarrow \mathfrak{e}_{0}(X)$ : $x \rightarrow\{x\}$ is a homeomorphism, and that $I(X)$ is closed in $\mathfrak{e}_{0}(X)$. Thus $X$ is locally compact.

Fell's topology on $\mathfrak{e}(X)$ may be described as that generated by two smaller topologies. The local topology has a sub-basis consisting of $\mathfrak{e}(X)$ and sets of the form $\{F \in \mathfrak{e}(X): F \cap N \neq \varnothing\}$, where $N$ is a nonempty open set in $X$. The global topology has as a sub-basis sets of the form $\{F \in \mathfrak{C}(X): F \subseteq P\}$, where $P$ is the complement of a compact proper subset of $X$. One verifies that for any $X$, if $F, F_{\alpha} \in \mathfrak{e}(X)$, then $F_{\alpha} \rightarrow F$ locally if and only if $F \subseteq \lim$ inf $F_{\alpha}$, and that if $X$ is Hausdorff and locally compact, $F_{\alpha} \rightarrow F$ globally if and only if $F \supseteq \lim \sup F_{\alpha}$.

\section{BIBLIOGRAPHY}

1. J. M. G. Fell, A Hausdorff topology for the closed subsets of a locally compact non-Hausdorff space, Proc. Amer. Math. Soc. 13 (1962), 472-476.

2. J. L. Kelley, General topology, Van Nostrand, New York, 1955.

3. C. Kuratowski, Topologie. I, 3rd ed., Monagrafie Mat. 20, Warsaw, 1952.

4. G. W. Mackey, Borel structures in groups and their duals, Trans. Amer. Math. Soc. 85 (1957), 134-165.

Columbia University 